\title{
Les agro-industries de l'interface métropolitaine Dakar-Thiès-Mbour : enjeux fonciers et développement territorial
}

\section{The agro-industries of the metropolitan interface linking Dakar, Thies and Mbour: Land stakes and territorial development}

\section{Las agroindustrias de la interfase metropolitana Dakar-Thies-Mbour: Cuestiones de bienes-raíces y desarrollo territorial}

\author{
Mame Cheikh NGOM, Sidia Diaouma BADIANE, Momar DIONGUE et Edmée \\ MBAYE
}

\section{Volume 61, numéro 172, avril 2017}

Version originale soumise en septembre 2016. Version révisée reçue en mai 2017.

URI : https://id.erudit.org/iderudit/1042714ar

DOI : https://doi.org/10.7202/1042714ar

Aller au sommaire du numéro

Éditeur(s)

Département de géographie de l’Université Laval

ISSN

0007-9766 (imprimé)

1708-8968 (numérique)

Découvrir la revue

Citer cet article

NGOM, M. C., BADIANE, S. D., DIONGUE, M. \& MBAYE, E. (2017). Les

agro-industries de l'interface métropolitaine Dakar-Thiès-Mbour : enjeux

fonciers et développement territorial. Cahiers de géographie du Québec, 61(172),

33-53. https://doi.org/10.7202/1042714ar

\section{Résumé de l'article}

Les espaces périurbains des grandes villes africaines font face aux mutations des systèmes agraires. Ils sont intégrés à l'économie de marché grâce à une production orientée vers la demande urbaine. La périphérie rurale de la métropole dakaroise est au coeur des dynamiques d'investissement par les agro-industries. L'article analyse les logiques d'implantation des entreprises agro-industrielles, les stratégies spatiales développées ainsi que leurs effets sur les communautés et l'environnement. L'analyse postule l'hypothèse de l'émergence d'un système de production agro-industriel porté par des acteurs de divers profils. Ces derniers s'inscrivent dans une logique capitaliste d'exploitation agricole, bouleversant l'organisation socioéconomique des populations locales ainsi que leurs rapports à l'espace. L'analyse s'appuie sur des données qualitatives recueillies en 2015 et 2017 auprès des entreprises agro-industriels et des populations de la zone. Les agro-industries, de profils variés, développent des stratégies territoriales inscrites dans une dynamique de production mondiale plus ou moins intégrée à la demande locale métropolitaine. 


\title{
Les agro-industries de l'interface métropolitaine Dakar-Thiès-Mbour: enjeux fonciers et développement territorial
}

\author{
The agro-industries of the metropolitan \\ interface linking Dakar, Thies and Mbour: \\ Land stakes and territorial development \\ Las agroindustrias de la interfase \\ metropolitana Dakar-Thies-Mbour: Cuestiones \\ de bienes-raíces y desarrollo territorial
}

\author{
Mame Cheikh NGOM, Sidia Diaouma BADIANE, \\ Momar DIONGUE et Edmée MBAYE \\ Département de Géographie \\ Faculté des Lettres et Sciences Humaines \\ Université Cheikh Anta Diop de Dakar (Sénégal) \\ thieeko@hotmail.com \\ sidia.badiane@gmail.com \\ dionguem@hotmail.com \\ edmeediouf@yahoo.fr
}

\section{Résumé}

Les espaces périurbains des grandes villes africaines font face aux mutations des systèmes agraires. Ils sont intégrés à l'économie de marché grâce à une production orientée vers la demande urbaine. La périphérie rurale de la métropole dakaroise est au cœur des dynamiques d'investissement par les agro-industries. L'article analyse les logiques d'implantation des entreprises agro-industrielles, les stratégies spatiales développées ainsi que leurs effets sur les communautés et l'environnement. L'analyse postule l'hypothèse de l'émergence d'un système de production agro-industriel porté par des acteurs de divers profils. Ces derniers s'inscrivent dans une logique capitaliste d'exploitation agricole, bouleversant l'organisation socioéconomique des populations locales ainsi que leurs rapports à l'espace. L'analyse s'appuie sur des données qualitatives recueillies en 2015 et 2017 auprès des entreprises agroindustriels et des populations de la zone. Les agro-industries, de profils variés, développent des stratégies territoriales inscrites dans une dynamique de production mondiale plus ou moins intégrée à la demande locale métropolitaine.

\section{Mots-clés}

Agro-industries, périphéries rurales métropolitaines, mutations foncières, territoire.

\begin{abstract}
The peri-urban areas of large African cities are experiencing a number of agricultural mutations. These areas are integrated into the market economy through a production system oriented towards meeting urban demand. The rural periphery of the metropolitan area of Dakar is at the core of these new investment dynamics, which are controlled by the agro-industries. This article analyzes the logic of the implementation of agro-industrial units, the spatial strategies that have been developed as a result, as well as their impact on communities and the environment. Our central hypothesis is built around an emerging agricultural system rooted in a capitalist exploitation of the local resources, which is upsetting the economic and social organization of the local population, and its relationship with place. We conducted a qualitative analysis of the data collected in 2015 and 2017 with the agro-industries and the local population. The diverse agro-industries are developing territorial strategies that are inscribed in a dynamic of world production, which is more or less integrated to the local metropolitan demand.
\end{abstract}

\section{Keywords}

Agro-industries, metropolitan rural peripheries, land mutations, territory. 


\section{Resumen}

Los espacios periféricos de las grandes ciudades africanas enfrentan transformaciones de los sistemas agrarios. Estos sistemas integran la economía de mercado gracias a una producción orientada por la demanda urbana. La periferia rural de la metrópolis de Dakar se encuentra al centro de los dinamismos inversores de los productores agro-industriales. El artículo analiza las lógicas de implantación de los agro-industriales, las estrategias espaciales realizadas, así como sus efectos sobre las comunidades y sobre el medio ambiente. El análisis postula la hipótesis de la manifestación de un sistema de producción agro-industrial sostenido por actores de diversas características. Éstos participan de una lógica capitalista de explotación agrícola que modifica la organización socio-económica de las poblaciones locales, así como su relación al espacio. El análisis se apoya en datos cualitativos compilados en 2015 y 2017 de agro-industriales y de poblaciones de la zona. Los agroindustriales, de rasgos diversos, implantan estrategias territoriales que corresponden a una dinámica de producción mundial, más o menos integrada a la demanda local metropolitana.

\section{Palabras claves}

Agroindustriales, periferias rurales metropolitanas, transformaciones de bienes-raíces, territorio.

\section{Introduction}

Dans le contexte de formation d'une aire métropolitaine interrégionale multipolaire, l'interface périurbaine Dakar-Thiès-Mbour est devenue un enjeu majeur de développement territorial. Elle est le territoire privilégié de déploiement des grands projets d'infrastructure de Dakar. Le pôle urbain de Diamniadio y est en cours d'aménagement depuis 2014 sur un périmètre de 1664 ha. L’aéroport international Blaise Diagne (AIBD), dont la construction a commencé en 2007, devra être fonctionnel en 2018. L'autoroute à péage Dakar-Diamniadio est ouverte à la circulation depuis 2013, et le tronçon Diamniadio-AIBD, depuis décembre 2016. Outre cette dynamique liée à de grands projets, cette interface connaît une nouvelle dynamique d'investissements agricoles modernes et intensifs.

Les mutations agricoles de cette interface périurbaine ont été étudiées par plusieurs auteurs (Arnaud, 1970; Dubresson, 1979; Bellot et Denis, 1989; Dione, 1989; Diagne, 1990; Fall et Fall, 2001 ; Bâ, 2007; Faye, 2007; Thiandoum, 2013). Ces auteurs se sont intéressés notamment à l'appropriation, à la transformation et à l'utilisation des ressources locales. Leurs analyses ont révélé les mutations des sociétés, des systèmes de production agricole et de gestion foncière, ainsi que des paysages de cette interface intégrée à l'économie de marché avec une production orientée vers la demande urbaine.

Toutefois, ces études présentent des limites. Les analyses sont descriptives. Elles ont caractérisé les systèmes de production, les circuits d'approvisionnement et les structures d'encadrement. La question de l'appropriation, de la transformation et de l'utilisation des ressources locales est appréhendée dans le cadre de rapports de domination entre citadins et ruraux. Ces études se sont intéressées aux activités agricoles en se focalisant sur le maraîchage, l'arboriculture ou l'aviculture. L’agro-industrie est étudiée comme un système de production agricole parmi d'autres, un élément d'une typologie. La question de l'aménagement et du développement territorial de l'interface est occultée. Or, dans le contexte actuel marqué par la décentralisation et la métropolisation, cette question constitue un enjeu majeur. 
La métropolisation dakaroise a jusqu'à maintenant épargné l'agro-industrie dans son déploiement en milieu périurbain, au détriment des autres activités agricoles. D’où notre choix de se focaliser sur l'agro-industrie qui, paradoxalement, enregistre une nouvelle dynamique de conquête spatiale dans l'interface métropolitaine dans un contexte marqué par une évolution des modalités d'accès au foncier. Quelles sont les stratégies d'acquisition foncière des agro-industries? Les aménagements agro-industriels s'inscrivent-ils dans une logique de production orientée vers le marché mondial au détriment de l'aire métropolitaine? Quels sont les effets des investissements agro-industriels sur l'aménagement et le développement des territoires des communautés locales?

Dans notre étude, nous postulons l'hypothèse de l'émergence d'un système de production agro-industriel porté par des acteurs de divers profils, et inscrit dans une logique capitaliste d'exploitation agricole qui bouleverse l'organisation socioéconomique des populations locales ainsi que leurs rapports à l'espace. Notre objectif est d'analyser les logiques d'implantation des unités agro-industrielles dans la périphérie métropolitaine dakaroise, les stratégies spatiales et territoriales utilisées, ainsi que leurs effets sur les communautés et l'environnement locaux.

L'étude s'appuie sur des données qualitatives collectées en 2015 et 2017 par une revue de documentation et la conduite d'entretiens auprès des agro-industries et des acteurs locaux des terroirs d'implantation des unités agro-industrielles. L'enquête de terrain comporte un travail d'observation pour documenter l'état de l'environnement et de la gestion des ressources naturelles, notamment le foncier.

Nous présentons d'abord le contexte et la démarche méthodologique. Ensuite, nous analysons les logiques d'implantation des unités agro-industrielles, ainsi que les stratégies spatiales et territoriales de ces acteurs économiques. Enfin, nous étudions les effets de ces activités sur les communautés et l'environnement local.

\section{Présentation de l'interface métrolitaine Dakar-Thiès-Mbour}

Le terrain d'étude est l'espace périurbain compris entre Dakar, Thiès et Mbour, qui sont les pôles urbains de l'aire métropolitaine. Cet espace couvre une superficie de $3852 \mathrm{~km}^{2}$ pour une population de 1739897 habitants en 2013 (ANSD, 2014) soit $13,5 \%$ de la population nationale. La densité moyenne de la population dans cette zone est de $453 \mathrm{hab} . / \mathrm{km}^{2}$ (ANAT, 2015). Les établissements humains du périurbain sont essentiellement constitués de villages et de petites villes comme Pout, Diamniadio, Sébikhotane, Kayar et Nguékhokh, qui ont un poids démographique compris en 8000 et 30000 habitants.

Cette zone présente trois principales caractéristiques physiques: une partie nord constituée d'un système dunaire et de sols hydromorphes, unité morphologique appelée Niayes, ${ }^{1}$ située au nord de Sangalkam (figure 1) ; une zone centre constituée de massifs, dont le horst de Diass et le plateau de Thiès; et une partie sud de l'interface métropolitaine marquée par l'existence de plage de sable fin entre Dakar et Mbour, zone communément appelée Petite Côte et fortement marquée par l'activité touristique, notamment internationale.

1 Les Niayes sont un système dunaire parallèle au cordon littoral et reliés par des dépressions interdunaires où affleure la nappe phréatique et y prédomine une végétation de type guinéenne. 


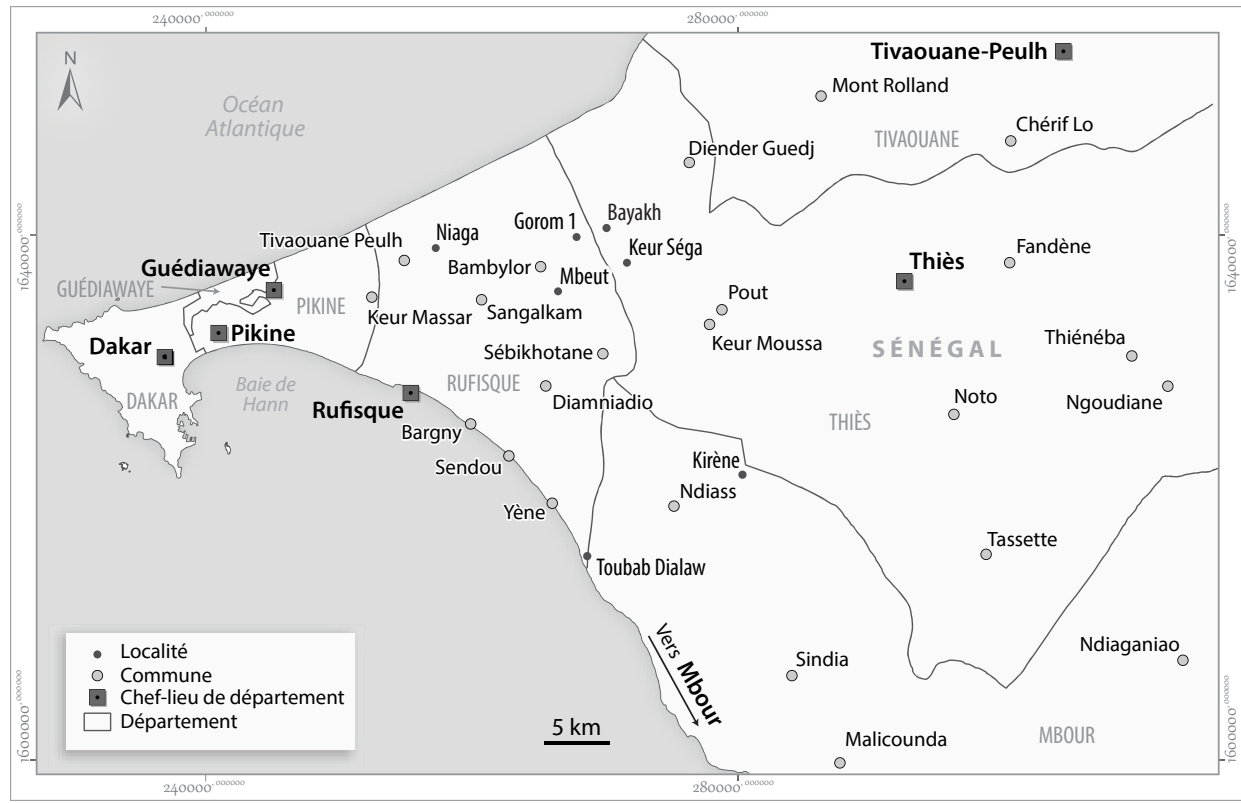

\section{L'agriculture dans l'interface métropolitaine: enjeux et approches}

Les transformations socioéconomiques, spatiales et agricoles induites par la ville dans l'interface métropolitaine Dakar-Thiès-Mbour ont très tôt retenu l'attention des géographes (Arnaud, 1970; Dubresson, 1979; Dione, 1986). Les conditions favorables à l'agriculture, l'entreprise de modernisation des techniques et de diversification des systèmes de production, les circuits d'approvisionnement et de commercialisation, les mutations paysagères et socioéconomiques de la société rurale, l'épineuse question foncière et l'avenir de cette entreprise favorisée par la proximité de la ville, mais aussi menacée par son avancée, sont les principales contributions de recherches portées par des géographes, des agronomes et des économistes (Bellot et Denis, 1989; Diagne, 1990 ; Fall et Fall, 2001 ; Bâ Diao, 2004 ; Bâ, 2007 ; Faye, 2007 ; Thiandoum, 2013).

\section{La diversité des systèmes de production: différentes trajectoires de modernisation agricole}

La diversité des systèmes de production agricole de l'interface métropolitaine est une réalité (Fall et Fall, 2001 ; Bâ, 2007), malgré son incapacité à se substituer aux importations de produits de grande consommation pour satisfaire la demande de la capitale sénégalaise, dans un contexte de dégradation et de raréfaction des ressources naturelles (Mbaye et al., 1999). Cette diversification, centrée sur le maraîchage, l'arboriculture et l'élevage intensif, notamment avicole, a favorisé l'apparition de trois systèmes de production, traduisant des voies diverses de modernisation. 
Les techniques de production reposent soit sur des outils modernes (système d'irrigation au goutte à goutte, tracteur, salariat), soit sur un mélange d'outils modernes et traditionnels. La corrélation entre la taille des exploitations et leur niveau de modernisation, d'une part, et le profil de l'exploitant, d'autre part, a permis d'établir une typologie des exploitations agricoles de cet espace. Il en ressort trois (Arnaud, 1970; Fall et Fall, 2001) ou quatre types d'exploitation agricole correspondant à autant de profils d'exploitants (Bellot et Denis, 1989). Le premier type est l'agro-industrie, qui dispose d'un encadrement et de techniques de production modernes, avec une production principalement destinée à l'exportation. Le second type renvoie aux "maraîchers du dimanche», citadins dakarois qui exploitent un verger où se mêlent arboriculture, maraîchage et aviculture. L'exploitation sert aussi de lieu de récréation. Le troisième type correspond aux maraîchers locaux dotés d'un appareil de production moderne sur une superficie d'environ 2,5 ha. La deuxième et la troisième catégories sont considérées comme des exploitations moyennes. Le dernier type est l'exploitation «paysanne» qui associe le maraîchage et des cultures vivrières pluviales sur de petites exploitations de moins de deux hectares : il s'agit de la petite exploitation utilisant des techniques de production et des systèmes de culture qui sont un mélange d'archaïsme et de modernisme.

Cette mutation des systèmes et des techniques de production montre le degré d'intégration et de dépendance à l'économie marchande des espaces ruraux proches des villes, ainsi que les effets de développement socioéconomique sur les populations locales de cette entreprise agroéconomique qui reste fragile et dominée par les citadins (Bellot et Denis, 1989). L'agriculture de l'interface Dakar-Thiès-Mbour est multifonctionnelle, avec une reconnaissance, par les acteurs, de ses fonctions de production et de ses fonctions alimentaire, sociale, économique et environnementale (Bâ, 2007). Sa préservation et celle de l'environnement constituent des enjeux (Bâ, 2007) surtout dans le contexte de métropolisation marqué par l'apparition de nouveaux acteurs dans cette interface.

Les dynamiques actuelles de cet espace rural se construisent autour d'enjeux urbains, environnementaux et territoriaux. L'interface est un enjeu urbain majeur en tant que territoire privilégié de déploiement de grands projets d’infrastructure de niveau métropolitain, comme l'aéroport AIBD et la zone économique spéciale qui le jouxte, le pôle urbain de Diamniadio (40 000 logements y sont prévus) et les infrastructures routières comme l'autoroute à péage qui va connecter Dakar-Thiès-Mbour. L'État veut faire de cet espace un levier de développement économique de l'aire métropolitaine grâce à l'aménagement de nouvelles centralités urbaines. Il est évident que ces grands projets urbains affectent l'environnement de l'interface, à cause de leurs méthodes modernes de culture. Les systèmes naturels, comme celui des Niayes, et les espaces agricoles et boisés subissent une forte pression liée à l'étalement urbain et à l'industrie extractive. L'agriculture périurbaine, notamment l'agro-industrie, pose problème sur le plan environnemental à cause de la compétition qu'elle exerce sur des ressources comme l'eau et la terre. À cela s'ajoutent les nuisances et les risques de pollution et de dégradation de l'environnement. Dans un contexte marqué par la décentralisation, le développement de ces différentes activités constitue un enjeu territorial pour les acteurs locaux. Ces derniers veulent développer leur territoire, qui est sollicité par plusieurs acteurs des activités urbaines, industrielles et agro-industrielles. Ces différents enjeux soulèvent la question de l'aménagement et du développement des territoires de l'interface. 


\section{L'agro-industrie dans l'interface: une approche par le développement territorial}

Dans le cadre de cette recherche, nous nous intéressons à l'agro-industrie. Ce choix est fondé sur le constat d'une situation paradoxale: là où les autres types d'exploitation agricole régressent, l'agro-industrie s'inscrit dans une dynamique d'expansion spatiale dans un contexte de métropolisation marqué par un développement sans précédent d'activités, d'infrastructures et de services supérieurs métropolitains dans l'espace périurbain situé entre Dakar, Thiès et Mbour (Diongue, 2012).

La définition de l'agro-industrie retenue ici se fonde sur le niveau de mécanisation et la taille de l'unité, l'organisation de la production, les capacités de production, le marché visé, la stratégie de gestion et le nombre d'emplois. L’agro-industrie caractérise une unité de production agricole qui utilise des outils sophistiqués de mécanisation pour une exploitation optimale des ressources (eau, sol, semences, intrants, matériel et main-d'œuvre) dans une logique capitaliste, sur un périmètre de vaste étendue (plus de 100 ha) nécessitant la mise en place d'un système d'organisation et de gestion moderne combinant le salariat et l'emploi d'une importante main-d'œuvre temporaire, de manière saisonnière ou journalière, pour favoriser une production intensive de végétaux (maraîchage, arboriculture) ou d’animaux destinés aux grands marchés urbains, nationaux ou mondiaux. L'activité de production agricole renvoie aux étapes nécessaires au déroulement, à la maîtrise et à l'exploitation d'un cycle biologique, végétal ou animal. Les activités exercées dans le prolongement de cette production, comme la transformation, le conditionnement, la conservation, le stockage et la vente des produits provenant de l'exploitation, sont également considérées agricoles, selon la loi nº 2004-16 du 4 juin 2004 portant sur l’Orientation agro-sylvo-pastorale.

Notre recherche s’intéresse spécifiquement aux agro-industries spécialisées dans la production maraîchère et arboricole. Dans cet article, nous privilégions une approche par le développement territorial qui met l'accent sur la notion de ressources territoriales (Pecqueur, 2005; Glon et Pecqueur, 2006). Pecqueur (2005) différencie les ressources données (naturelles) de celles construites (savoir-faire, services). Les premières renvoient aux espaces naturels valorisés (agricoles) et non mis en valeur. Les secondes prennent souvent une forme idéelle. La notion de ressource permet d'aborder les dimensions productive, environnementale et territoriale du développement. Les ressources de l'interface Dakar-Thiès-Mbour sont soumises à une forte pression métropolitaine (Diongue, 2012). Elles sont au cœur de logiques d'acteurs locaux, nationaux, voire mondiaux. L'analyse se focalise sur les enjeux locaux de développement de l'agro-industrie dans l'interface métropolitaine.

Le choix de cette approche se justifie par l'état de la recherche sur les activités agricoles dans la zone d'étude, d'une part, et par le contexte métropolitain actuel, d'autre part. Les recherches sur l'agriculture périurbaine dakaroise souffrent d'une faiblesse de la théorisation. Elles ont privilégié une approche descriptive qui a permis de caractériser les systèmes de production, les circuits d'approvisionnement et les structures d'encadrement. Elles ont appréhendé la question de l'appropriation, de la transformation et de l'utilisation des ressources locales dans le cadre de rapports de domination entre citadins et ruraux. Le rôle des acteurs publics locaux dans le processus d'appropriation du foncier est absent des analyses. L'agro-industrie est étudiée comme un système de production agricole parmi d'autres, un élément d'une typologie. La question de l'aménagement et du développement territorial de 
l'interface est occultée. Or, dans ce contexte actuel marqué par la décentralisation et la métropolisation, cette question constitue un enjeu majeur pour les acteurs locaux. Depuis 1996, les collectivités locales sont compétentes pour gérer le domaine national qui constitue l'essentiel du foncier agricole de l'interface. L'analyse de leur rôle dans l'installation des agro-industries s'impose, de même que leurs attentes en termes de développement local. Ce développement résulte d'une dynamique complexe entre acteurs privés et publics et une certaine forme de gouvernance (Leloup et al., 2003). Il exige une coordination verticale entre le local et le global, et horizontale entre acteurs, communautés et territoires. L'enjeu pour les acteurs locaux consiste à territorialiser l'agro-industrie, à l'intégrer dans une dynamique de développement local. Cela nous permet de mettre l'accent sur la place des communautés locales dans la dynamique d'implantation des agro-industries, mais aussi sur la gouvernance des ressources, notamment foncières, qui constitue un enjeu de développement territorial.

L'étude s’appuie sur des données qualitatives collectées en juillet 2015 et mars 2017 par une revue de documentation et la conduite d'entretiens auprès des acteurs agro-industriels et des acteurs locaux des terroirs d'implantation des unités agro-industrielles. L'enquête de terrain comporte un travail d'observation pour documenter l'état de l'environnement et de la gestion des ressources naturelles, notamment le foncier. Les enquêtes se sont déroulées dans les collectivités de Diass, de Sébikhotane, Keur Moussa et de Bambylor. Elles ont ciblé les acteurs locaux (élus, notables, responsables associatifs ou de coopératives de producteurs agricoles) et les acteurs agro-industriels. Le guide d'entretien adressé aux acteurs agro-industriels visait à caractériser l'unité agro-industrielle en mettant l'accent sur le projet d'implantation, l'acquisition foncière, les aménagements, les sources d'approvisionnement en eau, la production et sa (ou ses) destination(s), les relations avec les acteurs locaux, la perception de l'interface métropolitaine en lien avec les perspectives de développement de l'activité agricole.

L'enquête a ciblé les agro-industries spécialisées dans la production maraîchère et arboricole. Nous avons enquêté auprès des principales agro-industries à savoir SAFINA, Soleil Vert, Van Oers et Sénégal-Maroc-France (SEMAF). Ces industries ont toutes le statut d'entreprise franche d'exportation (EFE). ${ }^{2}$ SAFINA est une entreprise de la famille Filfili qui a démarré ses activités en 1989. Soleil Vert, entreprise de la famille Marruci, a été créée en juin 1999. Van Oers Sénégal, filiale de la multinationale néerlandaise du même nom, s'est installée en 2008. La SEMAF, mise en place par des partenaires sénégalais, marocains et français, a entamé ses activités en 2015. L’agro-industrie fait partie des facteurs exogènes de mutation de l'espace, à côté de l'industrie extractive et de l'urbanisation galopante dans cette zone. Les sociétés agro-industrielles qui se sont installées dans la zone occupent des superficies assez importantes, de l'ordre de dizaines à des centaines d'hectares: Société industrielle agroalimentaire (SIAGRO) (30 ha), Van Oers (600 ha), SAFINA (700 ha), Soleil Vert (130 ha), etc.

L'enquête dans les terroirs d'implantation des agro-industries, conduite auprès des notables, des élus locaux et des acteurs associatifs, avait trait aux aménagements agroindustriels et à leurs impacts sur les activités économiques, les pratiques spatiales et le cadre de vie dans ce secteur. Il s'agissait d'entretiens individuels semi-dirigés et de

2 Elles sont exonérées de la taxe sur la valeur ajoutée (TVA) sur les intrants et le matériel importé ainsi que sur l'exportation de la production. Elles peuvent en retour exporter $80 \%$ de leur production agricole. 
groupes de discussion. Dans le terroir d'implantation de Van Oers, Kirène, nous avons animé deux groupes de discussion. Le premier avec les responsables du Regroupement des producteurs maraîchers de Kirène (RPMK), qui fédère 40 groupements d'intérêt économique exploitant un périmètre agricole irrigué de 40 ha. L’objectif était de comprendre les relations entre le groupement de petits producteurs locaux et l'entreprise Van Oers: transfert de savoir-faire, appui matériel et technique, partage de calendrier cultural dans une perspective d'encadrement et d'intégration dans des filières de commercialisation de la production. Le second groupe de discussion rassemblait des notables, des élus locaux et des jeunes du village, soit au total 12 membres. Il visait à documenter l'impact de l'agro-industrie sur les communautés locales à travers le foncier, l'environnement, l'emploi et les services à la collectivité.

Ces données d'enquête ont fait l'objet d'une analyse thématique. Celle-ci visait, d'une part, à caractériser les unités agro-industrielles selon le statut juridique, les modalités d'accès au foncier, les aménagements agricoles et les types de production, ainsi que le marché et l'emploi. Elle a été complétée par une documentation des politiques agricoles et des programmes centrés sur la promotion des filières maraîchères et arboricoles. Un travail de localisation des unités agro-industrielles et d'observation des paysages a permis de cartographier la zone d'étude. D'autre part, l'analyse des résultats de l'enquête auprès des acteurs locaux a mis l'accent sur leur perception de l'agro-industrie en termes de possibilités, de risques ou de contraintes sur le terroir, de gestion foncière et de pratiques.

\section{L'agro-industrie: logiques, stratégies et impacts territoriaux}

Partant du projet pionnier et emblématique de BUD-Sénégal, société de maraîchage industrielle créée en 1972 et liquidée en 1979, notre analyse retrace le déploiement des unités agro-industrielles dans l'interface étudiée. L'enjeu consiste à montrer le changement de logiques d'implantation des unités agro-industrielles, avec le passage d'une logique d'installation promue par l'État à une logique privée, portée par des acteurs nationaux et internationaux, où les collectivités locales jouent un rôle dans l'accès au foncier agricole. Nous nous focalisons ensuite sur les stratégies spatiales et territoriales des agro-industries. Enfin, dans la dernière partie, nous nous interrogeons sur les impacts des agro-industries sur les communautés et l'environnement local.

\section{L'accès au foncier des agro-industries: de l'intervention étatique aux stratégies individuelles d'accès}

Jusqu'en 1964, deux régimes de tenure foncière prédominaient au Sénégal: le droit foncier africain précolonial et le droit colonial. Le premier conçoit la terre comme un bien communautaire, sacré et inaliénable. Le second reconnaît l'écrit et la mise en valeur comme principales sources de justification de la propriété foncière, qui est individualisée.

La loi 64-46 du 17 juin 1964 relative au Domaine National considère l'État comme l'unique détenteur des terres non immatriculées à son nom ou pour le compte d'une autre personne, physique ou morale, avant son entrée en vigueur. Le domaine national comprend quatre catégories de zones: la zone urbaine, qui correspond au périmètre d'intervention des communes ou des groupements d'urbanisme; la zone classée à vocation forestière ou de protection; la zone de terroir, destinée 
à l'habitat rural, la culture ou l'élevage; enfin, la zone pionnière, qui a vocation d'accueillir des projets de développement ou d'aménagement. L’État reconnaît un droit d'usage sur le domaine national qui ne relève d'aucune propriété. Seul l'État a le droit d'immatriculer les terres du domaine national en les intégrant dans son domaine privé, qu'il peut concéder sous la forme d'un permis d'occuper, d'un bail ordinaire, d'un bail emphytéotique, d'un droit de superficie ou d'une vente.

Ce régime foncier ne reconnaît pas la propriété coutumière. Cette situation est à l'origine de la cohabitation de deux régimes juridiques fonciers au Sénégal: traditionnel et moderne. Une telle dualité des régimes explique l'existence de conflits d'appropriation de la terre où la légitimité est opposée à la légalité (Crousse et al., 1986).

Dans le processus d'approfondissement de sa politique de décentralisation, l'État a transféré la gestion du domaine national aux collectivités locales par l'intermédiaire de la loi 96-07 du 22 mars 1996. Les terres de l'interface métropolitaine relèvent essentiellement du domaine national. L'implantation des agro-industries dans l'interface s'inscrivait, au départ, dans une logique étatique de promotion de l'économie maraîchère. Depuis 1996, elle s'inscrit dans des logiques propres aux agro-industries.

\section{Les logiques d'implantation des unités agro-industrielles}

Le phénomène d'accaparement des terres de culture et des pistes de parcours du bétail, observé dans l'aire métropolitaine en gestation, amorce l'aménagement d'un nouveau pôle urbain, mais pose des problèmes qui obéissent à des logiques d'implantation qu'on peut mettre en corrélation avec la situation de la paysannerie de l'époque coloniale, avec l'interventionnisme de l'État et avec l'implantation des agro-industries.

\section{L'intervention étatique: une logique d'appui à l'implantation dans le cadre de zones pionnières}

À l’image de beaucoup de pays en développement, le Sénégal a misé sur le développement de son agriculture pour donner une impulsion à sa croissance économique. Ainsi, depuis son accession à l'indépendance, des politiques et programmes ont été mis en œuvre pour moderniser l'agriculture. L'interface métropolitaine s'est distinguée par la création de deux zones pionnières en 1971, Baobab (700 ha) et Kirène (180 ha), pour l'implantation du projet maraîcher de la société BUD-Sénégal.

Au début des années 1970, le projet BUD-Sénégal est la première initiative de promotion de l'agro-industrie dans la périphérie de l'aire métropolitaine dakaroise. Ce projet de modernisation agricole s'inscrit alors dans une politique d'amélioration des conditions de production et de commercialisation des produits maraîchers au Sénégal. Les enjeux de son implantation consistent à faire du Sénégal un grand exportateur de produits maraîchers vers l'Europe, d'une part, et à servir de levier pour moderniser des exploitations traditionnelles par l'assistance et la vulgarisation de nouvelles techniques, d'autre part. L'agro-industrie devait permettre de sortir de l'opposition entre une agriculture utilisant des techniques modernes et celle marquée par l'archaïsme des techniques de production (Dione, 1986). 
Filiale d'un grand producteur de légumes au niveau mondial, BUD-Sénégal est créée par le décret 72-138 du 21 février 1972. L'entreprise utilise des techniques de production très sophistiquées ${ }^{3}$ dans ses deux exploitations (Baobab et Kirène) et un personnel d'encadrement constitué d'expatriés et de nationaux (Dione, 1986). La tomate, les poivrons, les haricots verts et les melons sont ses principales productions.

Toutefois, le projet BUD-Sénégal s'avère un échec, et ce, à cause de trois contraintes majeures (Dione, 1986: 111). Les contraintes pédologiques et hydriques du milieu physique sont responsables d'une chute des rendements. Le transport et la commercialisation de la production constituent une difficulté de taille. Si le marché européen est la principale cible de BUD-Sénégal, l'entreprise n’y écoule que $60 \%$ de sa production en raison de problèmes logistiques (fret à perte, coût élevé du transport par avion, faible capacité de conservation des produits). Elle doit se contenter du marché local (40\% de la production) pour minimiser ses pertes. Enfin, la mauvaise gestion de BUD-Sénégal est responsable d'un déficit de plus de 700 millions de francs de la Communauté financière en Afrique (CFA). Face au désengagement des actionnaires privés, l'État sénégalais prend la relève en faisant passer de $48 \%$ à $61 \%$ son capital-actions dans l'entreprise. Toutefois, le manque d'expérience de la nouvelle équipe accentue la crise et, le 3 septembre 1979, l'État déclare la compagnie en faillite (Dione, 1986: 113).

Pour continuer à exploiter les sites, l'État a financé Sénégal Périmètre Maraîcher (SENPRIM), composé d'une trentaine de groupements paysans. L'échec de SENPRIM à son tour, en 1989, se traduit par l'installation de l'agro-industrie SAFINA sur le périmètre de Baobab, de l'ex-BUD-Sénégal. Aujourd'hui, SAFINA exploite 700 ha de la zone pionnière de Baobab. L'entreprise Van Oers exploite, rien que dans la zone pionnière de Kirène, 13 ha de terre. Toutefois, ces agro-industries ne se limitent pas aux périmètres des zones pionnières. Elles développent des logiques d'accès au foncier agricole par le canal des pouvoirs publics locaux et des propriétaires coutumiers.

\section{Des nouvelles logiques d'accès aux fonciers des agro-industries}

Les agro-industries développent des logiques privées d'accès au foncier agricole en recourant à la location de terres et à des acquisitions auprès des acteurs locaux.

Outre son périmètre de Baobab, SAFINA exploite 300 ha sur le domaine national dans la commune de Bambylor. De plus, elle revendique un titre foncier dans la collectivité locale de Keur Moussa. ${ }^{4}$ Van Oers exploite, au début, 600 ha qui sont essentiellement constitués des terres du domaine national que la collectivité locale de Ndiass lui a affectés: "Van Oers a acquis l'hectare à 200000 francs CFA [300 €] auprès des propriétaires coutumiers » (Entretien: notable du village de Kirène, 07 /07/15). Soleil Vert a démarré ses activités en 1999 en louant 76,05 ha auprès d'un entrepreneur local, Gora LEYE. Elle s'est progressivement inscrite dans une stratégie d'acquisition foncière auprès de ce dernier et des propriétaires coutumiers : «Le périmètre agricole

3 Irrigation au goutte à goutte, arrosage par aspersion, méthode gravitaire, station météorologique, piste d'atterrissage pour les avions assurant les traitements phytosanitaires, magasins de conditionnement, matériel agricole lourd, parc automobile important, etc.

4 Un conflit vieux de 20 ans oppose l'entreprise SAFINA aux propriétaires coutumiers de Keur Moussa. Durant le mois de janvier 2017, les jeunes de Keur Moussa ont manifesté leur opposition à l'extension de l'agro-industrie en saccageant les locaux de la commune de Keur Moussa. Ils reprochent à l'équipe municipale d'avoir délibéré et affecté une partie des terres du village à SAFINA. 
fait 130 ha. Soleil Vert est propriétaire de 80 ha. Le reste est en location. Soleil Vert a récemment vendu 20 ha à un blanc qui s'active dans la production de banane et la pisciculture» (Entretien: responsable du périmètre, 16/03/17). Ces agro-industries ont réussi à sécuriser leur périmètre en obtenant un bail auprès de l'État. La SEMAF, qui a récemment démarré ses activités, a loué tout son périmètre de 105 ha «à un grand commerçant qui réside à Dakar», selon un technicien agricole du site.

Les agro-industries de l'interface sont de moins en moins tournées vers l'État dans leurs stratégies d'acquisition foncière. Elles sont dans une logique privée d'appropriation foncière en s'adressant directement aux acteurs locaux, propriétaires coutumiers et collectivité locale. L'alliance entre agro-industrie et collectivité locale favorise ces acquisitions. Les périmètres agricoles de Van Oers, SAFINA et Soleil Vert prennent la forme d'enclaves territoriales clôturées donnant l'aspect d'un bocage avec un dispositif d'accès sécurisé.

\section{Les stratégies spatiales et territoriales des agro-industries}

Dans leurs stratégies d'implantation spatiales, les agro-industries exploitent les effets de la métropolisation. Il s'agit de la présence d’un marché local métropolitain en développement très porteur, d'une part, et des grandes infrastructures (port, aéroport et route) pour se positionner sur le marché mondial, d'autre part.

Non seulement le marché métropolitain dakarois est en développement, mais il est très compétitif pour certaines productions comme le melon, la tomate, voire les oignons. Les agro-industries de l'interface se positionnent sur ces produits pour approvisionner le marché local métropolitain.

La présence du port, de l'aéroport et des grands axes routiers constitue un atout sur le plan logistique pour accéder au marché international. La position stratégique du Sénégal favorise les agro-industries de l'interface Dakar-Thiès-Mbour, compte tenu de la proximité du marché européen. Le pays a des possibilités et des avantages comparatifs en matière d'approvisionnement des marchés des pays du Nord, surtout l'hiver. C'est le cas de Van Oers, de Soleil Vert, de SAFINA et de la SEMAF, qui exportent l'essentiel de leur production en Europe. Le choix d'écouler la production par le port ou l'aéroport dépend aussi de stratégies commerciales. En cas de saturation du marché européen, Soleil Vert privilégie l’affrètement par le port, jouant ainsi sur le temps (une semaine) pour espérer une conjoncture plus favorable.

L’interface métropolitaine répond aux stratégies spatiales des agro-industries dans la mesure où elle dispose de réserves foncières, de ressources en eau, de main-d'œuvre et d'infrastructures logistiques. Par ailleurs, dans un avenir proche, l'AIBD pourrait renforcer la compétitivité de ces entreprises en réduisant les coûts de fret.

La géographie du Sénégal présente des dispositions toutes particulières parce qu'elle offre des possibilités dans le cadre des échanges avec les pays d'Amérique et d'Europe. Cela se traduit par le développement de filières agricoles généralement dynamiques en période hors saison (après la saison des pluies au Sénégal). Ainsi, l'interface Dakar-Thiès-Mbour (figure 2) attire de plus en plus certains agroindustriels, dont Van Oers qui exporte ses productions au Pays-Bas. 
Figure 2 Localisation des périmètres agro-industriels dans l'interface Dakar-Thiès-Mbour

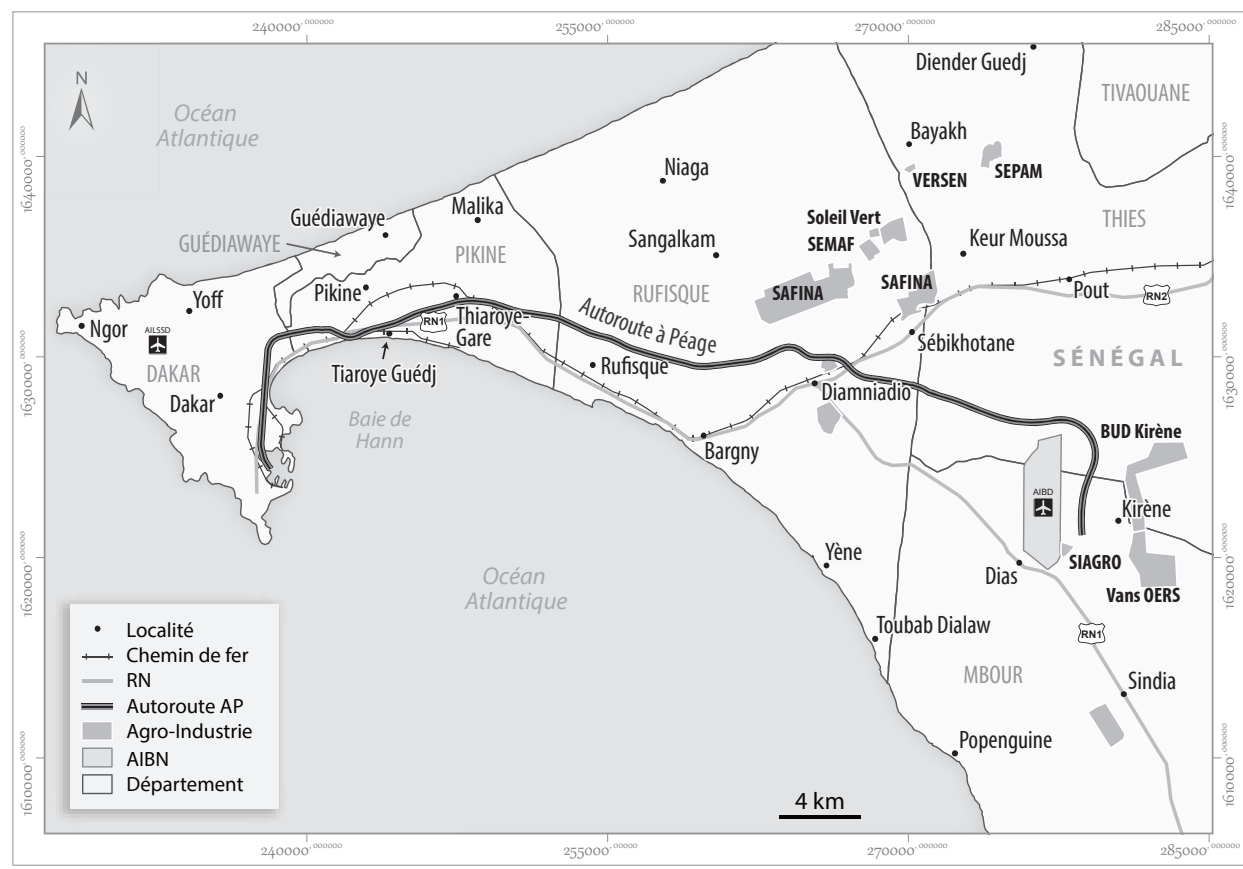

Conception: Ngom, Badiane, Diongue et Mbaye, 2017 Source:DTGC, 2014

\section{Système de production, culture et marché des agro-industries de l'interface}

Les agro-industries SAFINA, Soleil Vert, Van Oers et SEMAF, respectivement originaires du Sénégal, du Maroc et de la France, utilisent des techniques modernes de production agricole basées sur la mécanisation et les systèmes d'irrigation de goutte à goutte. SAFINA exploite cinq forages dans la zone de Sébikhotane (située à $30 \mathrm{~km}$ de Dakar) : trois d'une capacité de $250 \mathrm{~m}^{3}$ /heure et deux de $150 \mathrm{~m}^{3}$ /heure. Soleil Vert exploite un forage de $150 \mathrm{~m}^{3}$ /heure et Van Oers en utilise aussi deux de même capacité. Ces agro-industries maîtrisent leur approvisionnement en eau.

Ces quatre entreprises se spécialisent dans la production de trois, voire quatre, denrées (tableau 1). Le haricot vert est cultivé par les quatre. Avec le melon, il constitue la principale production maraîchère de SAFINA et de Soleil Vert, qui disposent aussi de vergers de manguiers. Van Oers cultive le maïs doux et l'oignon vert, en plus du haricot vert. La SEMAF a quatre productions : haricot vert, maïs doux, tomate et papaye. 
Tableau 1 Cultures des agro-industries Soleil Vert et SEMAF

\begin{tabular}{c|c|c|c|c|c}
\hline Entreprise & Culture & Superficie (ha) & Variété & $\begin{array}{c}\text { Rendement } \\
\text { en tonne/ha }\end{array}$ & $\begin{array}{c}\text { Calendrier de } \\
\text { production }\end{array}$ \\
\hline \multirow{4}{*}{ Soleil Vert } & Melon & 50 & Charentais & 20 & Septembre à mars \\
\cline { 2 - 6 } & Haricot vert & $80-100$ & $\begin{array}{c}\text { Filet } \\
\text { Bobby }\end{array}$ & $\begin{array}{c}5-10 \\
8-15\end{array}$ & Novembre à avril \\
\cline { 2 - 6 } & Mangue & 24 & Kent et Keit & - & Mi-mai à début août \\
\hline \multirow{5}{*}{ SEMAF } & Maïs doux & 80 & Étrangère & 10 & - \\
\cline { 2 - 6 } & Haricot vert & 40 & Bobby et Filet & - & Novembre à avril \\
\cline { 2 - 6 } & Tomate & 20 & Locale & 60 & Mars à août \\
\cline { 2 - 6 } & Papaye & 5 & Locale & 20 & Septembre à août \\
\hline
\end{tabular}

Conception: Ngom, Badiane, Diongue et Mbaye, 2017

Source: Enquête de terrain menée auprès des agro-industries, mars 2017

Les techniques de production de ces agro-industries répondent aux normes de production de qualité et de traçabilité des partenaires commerciaux surtout européens. Elles ont la certification Globalgap. Van Oers Sénégal profite du savoir, des compétences techniques, du mode de gestion standardisée et des normes de production de son groupe, Van Oers United, pour se conformer aux exigences du marché européen. Elle maîtrise toute la chaîne de production maraîchère grâce à une recherche-développement sur les semences de ses produits stratégiques, les traitements, les systèmes d'irrigation et la fertilisation des sols (figure 3). Ce type d'agro-industrie n'a pas besoin d'une assistance technique et organisationnelle en dehors de son groupe. Soleil Vert a des partenaires européens qui lui fournissent les semences de haricot (Bobby, Rivergaro, Filet Alexandra) et de melon (Lisse, Ecrit).

\section{Figure 3 Culture de maïs doux dans le périmètre agro-industriel de Van 0ers, Kirène, juillet 2015}

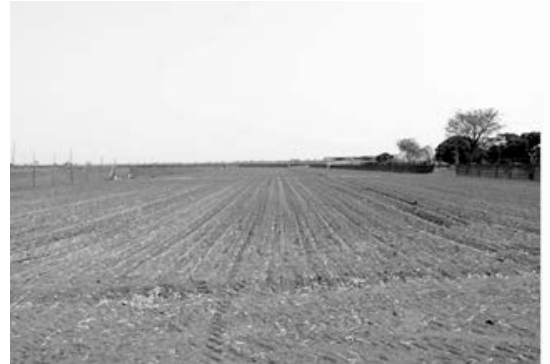

Source: Ngom, Badiane, Diongue et Mbaye, 2015

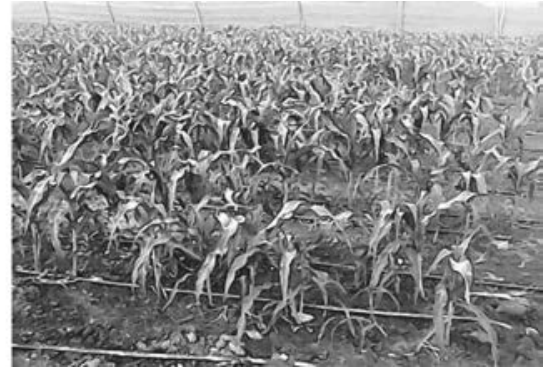

Ces agro-industries recourent à une importante main-d'œuvre dans la production, le désherbage, la récolte et le conditionnement du haricot, du melon, de la tomate et de la mangue (figure 4). Soleil Vert fait appel à une main-d'œuvre constituée de permanents et de saisonniers. La main-d'œuvre permanente est issue de la zone, et la saisonnière est recrutée dans un rayon de 20 à $60 \mathrm{~km}$. Le nombre de femmes qui s'activent dans le tri (les trieuses) varie de 150 à 300 . 
La main-d'œuvre permanente est issue de la zone dans un rayon de moins de $10 \mathrm{~km}$. Le personnel d'encadrement est sénégalais. La main-d'œuvre temporaire est recrutée dans un rayon de 20 à $60 \mathrm{~km}$. Elle est utilisée dans les activités de désherbage et de récolte. Ces activités n'attirent pas les gens de la zone à cause de la faible rémunération du travail et des conditions de travail qui sont dures. C'est pourquoi les femmes de la zone préfèrent les activités de tri. La main-d'œuvre temporaire est payée à la tâche. La récolte d'un seau de $5 \mathrm{~kg}$ de haricot est payée à 200 francs CFA (30 centimes d'euro). S'agissant du melon, le paiement se fait à la journée soit 2500 francs CFA $(3,8 €)$. Dans le périmètre de production, la main-d'œuvre saisonnière de sexe masculin est constituée d'étrangers, Guinéens, Burkinabés (Entretien: responsable du périmètre de Soleil Vert, 16/03/17).

\section{Figure 4 Récolte de mangues dans le périmètre agro-industriel de SAFINA, Sébikhotane, juillet 2015}
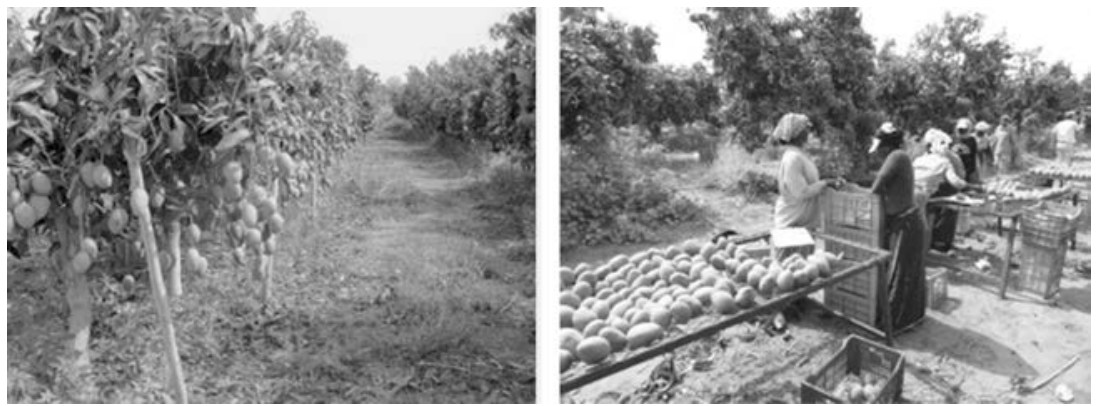

Source: Ngom, Badiane, Diongue et Mbaye, 2015

Van Oers utilise de la main-d'œuvre locale, en majorité des femmes (figure 5). Si les hommes se distinguent dans l'agriculture, les femmes s'activent dans la récolte, le tri, le conditionnement, le pesage et l'emballage. Elles sont des milliers, réparties en fonction de leur statut: il s'agit des caporales (1\%), des surveillantes (5\%), des peseuses (7\%), des trieuses (25\%) et des récolteuses (62\%). Leur temps de travail est de huit heures, mais elles font beaucoup d'heures supplémentaires. «Il nous arrive de travailler jusqu'à minuit ou une heure du matin. Même si le transport à l'aller et au retour est assuré par le car de ramassage, la gestion de nos foyers, de nos maris et de nos enfants est souvent compromise par les activités nocturnes. D’autre part, nous ne dormons pas assez pour revenir au champ à 8 heures » (Entretien: femme travaillant à Van Oers, 07/09/17 - hors saison).

Par rapport à la main-d'œuvre, les agro-industries participent au renforcement de l'expertise locale. Des jeunes ont appris à labourer avec des tracteurs, à pratiquer l'agriculture moderne et à utiliser des pesticides et autres intrants. Ainsi, ils servent de conseillers à leurs parents qui continuent de pratiquer l'agriculture traditionnelle. Cependant, le personnel saisonnier déplore l'arrêt des activités au mois d'avril : «En vérité, le champ nous aide à régler certains de nos besoins, mais le temps d'arrêt est trop long, c'est-à-dire du mois de mai au mois de décembre. Van Oers ne fonctionne que cinq mois [décembre à avril] alors que, dans nos villages respectifs, nos revenus par ménage sont trop faibles» (Entretien: habitant du village de Bandia, mitoyen à Van Oers, 10/09/17). 


\section{Figure 5 Les femmes trieuses du périmètre maraîcher de Van Oers, février 2017}
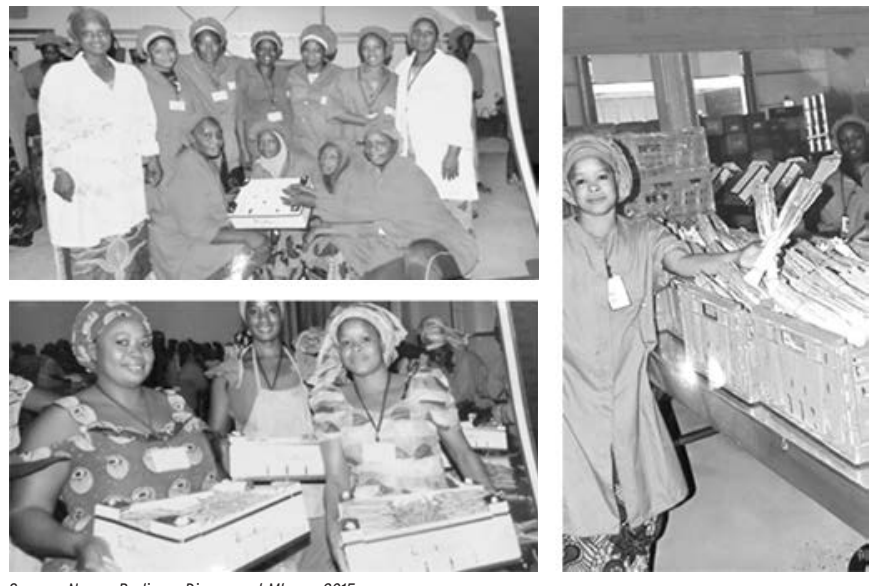

Source: Ngom, Badiane, Diongue et Mbaye, 2015

Les agro-industries dépendent plus ou moins de grands groupes étrangers notamment français qui, par ailleurs, imposent leur logique. Elles sont dans des rapports de force avec leur partenaire étrangers en essayant de maîtriser aux mieux les contraintes des marchés locaux et étrangers.

Van Oers, Soleil Vert, SAFINA et SEMAF sont à la fois sur le marché mondial et sur le marché métropolitain de Dakar. Toutefois, les productions de Van Oers sont plus destinées à l'étranger alors que SAFINA se positionne dans le marché local à travers une chaîne de supermarchés. En effet, les stratégies des agro-industries consistent à assurer un mode commercial combinant ces deux marchés.

Pour être présentes sur le marché européen, elles profitent de la période d'exploitation qui est favorable à la production sénégalaise, surtout en période d'hiver. Certaines ont des partenaires commerciaux sur le marché européen. C’est le cas de Soleil Vert et de Boyer SA (deuxième producteur de melon en France) : "Nous produisons du melon haut de gamme, notamment le Philibon qui est le produit phare de Boyer. L'épicerie fine constitue notre marché en France. Elle vise les catégories sociales aisées » (Entretien: responsables du périmètre de Soleil Vert, 16/03/17). Cette société française fournit tous les intrants et aussi le responsable de la production: «S'agissant du melon, tous les intrants sont importés" (Entretien: responsable conditionnement de Soleil Vert, 16/03/17). Soleil Vert est le premier exportateur de melon des agro-industries de l'interface (tableau 2). Sa production exportée est passée de 1800 tonnes en 2016 à 2200 en 2017. Toutes productions confondues, l'entreprise a exporté 3700 tonnes sur le marché européen, en 2016, dont 1200 tonnes de légumes et 2500 tonnes de fruits. 
Tableau 2 Les exportations de l'agro-industrie Soleil Vert

\begin{tabular}{c|c|c|c|c}
\hline \multirow{2}{*}{ Agro-industrie } & Culture & Part exportation & $\begin{array}{c}\text { Tonnage exporté } \\
\text { en 2016 }\end{array}$ & Pays d'exportation \\
\hline \multirow{3}{*}{ Soleil Vert } & Melon & $60 \%$ & 1800 & France \\
\cline { 2 - 5 } & Haricot vert & 50 à $60 \%$ & 1200 & $\begin{array}{c}\text { France }(700 \mathrm{t}) \\
\text { Pays-Bas }(500 \mathrm{t})\end{array}$ \\
\cline { 2 - 5 } & Mangue & $100 \%$ & 1700 & France \\
\hline
\end{tabular}

Conception: Ngom, Badiane, Diongue et Mbaye, 2017

Source: Enquête de terrain menée auprès des agro-industries, mars 2017

La SEMAF exporte le maïs doux et le haricot vert sur le marché européen par l'intermédiaire d'un partenaire commercial: «Nous avons jumelé avec une société française, Sweet Corn Midi-Pyrénées, qui s'occupe de l'écoulement de notre production de maïs doux» (Entretien: technicien agricole du périmètre de SEMAF, 16/03/17).

\section{Impacts de l'agro-industrie dans l'interface Dakar-Thiès-Mbour}

L’installation des sociétés agro-industrielles dans l'aire métropolitaine Dakar-Thiès-Mbour a des impacts environnementaux, fonciers et socioéconomiques.

\section{Impacts environnementaux de l'activité agro-industrielle}

L'analyse des impacts environnementaux se focalise sur certains éléments, notamment le déclassement des forêts, l'utilisation des produits chimiques, la dégradation des sols, l'exploitation des nappes et les rejets.

Les espaces naturels ont subi des pressions multiples, surtout d'ordre anthropique en raison d'activités industrielles. Ces activités, y compris celles de l'agro-industrie, ont occasionné le déclassement de certaines forêts. Plusieurs hectares de terre, précédemment affectés à des tiers pour l'arboriculture, sont déboisés après délibération du Conseil rural.

Pour la préparation des sites, il faut procéder à une coupe systématique des arbres, puis à un dessouchement. Les terrains sont ainsi mis à nu pour la culture. La conséquence est la perte d'espèces végétales et l'accélération de l'érosion des sols par les écoulements de surface et par le vent.

\footnotetext{
Nos terres de culture sont confisquées par les autorités locales au profit de la [société à responsabilité limitée] SARL Van Oers suivant une délibération en date du 26 octobre 2007. Je n'ai reçu en compense que 700000 francs CFA [l'équivalent de $1070 €$ ] pour un champ de 4 ha. Dès lors, je n'arrive plus à cultiver en période d'hivernage, je n'ai plus là où pratiquer la jachère pâturée, et les plantes médicinales ont disparu. La réalisation de ce projet agricole m'a rendu pauvre, car les autres terres qui me restent sont impraticables à cause des pesticides et de la poussière provenant du grand périmètre (Entretien: paysan du village de Séssène, situé à 1 km de Van Oers, 10/09/17).
}

Les périmètres agricoles sont clôturés avec des barbelés. Cela provoque une rupture des parcours du bétail et réduit sa mobilité, ce qui accentue les conflits entre agriculteurs et éleveurs. L'insuffisance ou le confinement des espaces de pâturage font que les éleveurs trouvent comme alternative l'émondage des arbres pour l'alimentation du bétail. Cette pratique a un impact sur la végétation, notamment sur les espèces prisées par les animaux. 
L'effet des produits chimiques (engrais, herbicides, etc.) peut aussi être souligné. L'agro-industrie nécessite l'utilisation de divers produits phytosanitaires pouvant avoir des effets réels sur les employés et sur l'environnement. Même si des mesures de protection sont prises, les employés restent exposés au risque d'inhalation des produits chimiques et toxiques. De plus, les sols ainsi que les eaux de surface et souterraines peuvent être affectés par les intrants chimiques.

\begin{abstract}
Au mois d'août de l'année 2016, les ouvriers chinois qui construisent l'autoroute à péage aperçoivent aux alentours de Van Oers un troupeau de bœufs en agonie. Ils ont aussitôt alerté les villageois de Dobour. Arrivé sur le lieu du drame, je me rends compte qu'il s'agit bien de mes 50 têtes de bœufs en quête de pitance. Nous avons pris contact avec un vétérinaire qui, après autopsie, révèle qu'ils ont brouté l'herbe entachée de pesticides. Soutenu par un membre de forum civil mondial, je cours toujours derrière cette affaire pour que justice soit faite. Mais nous sommes dans un pays où le dialogue ne vous oblige pas à attaquer directement l'agro-industrie sous prétexte que nos filles et nos fils perdent leurs emplois. Je suis passif devant cette affaire douloureuse et déboussolé (Entretien: paysan de Dobour, hameau villageois jouxtant le périmètre maraîcher de Van Oers, 15/09/17).
\end{abstract}

On observe aussi une pollution liée à la circulation automobile dans les pistes reliant les entreprises aux routes nationales RN1 (Van Oers, SIAGRO) et RN2 (SAFINA). Pour les poussières, la source d'émission actuelle est essentiellement liée au milieu naturel (envol). Il s’agit des effets des émissions de poussière sur le site (parcours non bitumés).

\title{
Impacts fonciers
}

L'espace périurbain Dakar-Thiès-Mbour est en passe de devenir un pôle de développement économique majeur. La question foncière est au cœur du processus de développement de ce secteur, qui change progressivement de vocation. Les évolutions récentes vont avoir comme conséquences, sur les systèmes naturels, la modification des modes d'occupation du sol.

Les affectations de terres à ces agro-industries ont souvent posé des problèmes en raison des difficultés d'accès à la ressource, de plus en plus ressenties par les populations. Les terres de culture rétrécissent à cause de la spéculation foncière provoquée, en partie, par l'installation des entreprises agro-industrielles.

Les deux périmètres (Baobab et Kirène) de l'ex-BUD-Sénégal peuvent servir d'illustration d'une expropriation foncière des populations au profit d'une agroindustrie. En effet, le site du projet BUD-Sénégal a été initialement classé «zone pionnière», en 1971, et attribué à des investisseurs américains.

L'accaparement de terres agricoles est alors manifeste et on assiste au remplacement progressif des systèmes traditionnels de production par des systèmes modernes, et donc à leur disparition. L'installation des multinationales a conduit à une désorganisation des terroirs et a imposé une recomposition spatiale. Il y a une tendance à la concentration du foncier agricole entre les mains des agro-industries de l'interface Dakar-Thiès-Mbour. Le manque de sécurisation du foncier agricole expose les populations à des risques d'expropriation foncière, ce qui les oblige à céder leurs terres, ou plutôt leur droit d'usage, au plus offrant. 
Mais les paysans ayant perdu leurs terres ne se laissent pas faire. Ils réclament à la commune et à Van Oers le respect des clauses du mémorandum signé à Diass, le 30 août 2007. Après la mise sur pied du Collectif des paysans pour la défense de leurs terres (CPDT), un procès-verbal de constat réalisé par l'étude de maître Cheikh Tidiane Tambadou (huissier de justice à Dakar) ouvre la voie à une poursuite judiciaire.

\section{Impacts socioéconomiques}

L’implantation d'agro-industries présente des avantages notables, mais aussi des inconvénients du point de vue socioéconomique. Même si leur mode d'implantation est décrié, les agro-industries demeurent de véritables pourvoyeuses d'emplois, surtout en milieu rural où les populations nourrissent souvent beaucoup d'espoir quand il s'agit d'un projet qui peut contribuer à l'amélioration de leur niveau de vie.

De plus, les entreprises agro-industrielles soutiennent les initiatives de développement communautaire, dans le cadre de leur responsabilité sociétale. Ainsi, la question de l'emploi occupe une bonne place dans le protocole qui lie Van Oers à l'ex-communauté rurale de Diass.

En effet, Van Oers emploie la main-d'œuvre locale, réduisant ainsi le chômage dans les villages. Sène (2013) affirme que 97\% de la main-d'œuvre de cette entreprise est locale. Le statut des employés est variable (permanents, contractuels, journaliers). Le salaire mensuel moyen varie entre 37500 et 60000 francs CFA pour les saisonniers et est de 80000 francs CFA pour les permanents. Cette main-d'œuvre est essentiellement féminine. D’après les personnes interviewées, Van Oers a favorisé le retour de plusieurs jeunes dans des villages comme Kirène, Diass, Toglou et Bandia. SAFINA offre beaucoup d'emplois saisonniers au niveau local. Cette entreprise a contribué à améliorer le niveau de vie des employés. Selon certains, leur emploi leur a permis de subvenir correctement aux besoins de leur famille et, mieux, de se déployer dans d'autres secteurs par un investissement personnel. Quoique mitigé, l'impact salarial est réel dans les villages autour des périmètres agro-industriels, voire au-delà.

Par ailleurs, diverses réalisations sont à mettre au crédit des entreprises dans les communautés. Il s'agit, notamment, de la construction de forages et de bien d'autres infrastructures et équipements sociaux de base (cases de santé). Les entreprises octroient également des fournitures scolaires aux écoles et des bourses d'étude. Un appui matériel est aussi accordé aux associations sportives et culturelles.

Les entreprises ont sensiblement influencé les systèmes de culture. Les paysans s'orientent davantage vers une modernisation. L'arboriculture a pris le dessus sur les autres productions traditionnelles. Ciss (2012), cité par Thiandoum (2013), souligne le fait que le complexe agro-industriel SAFINA a stimulé le développement de l'arboriculture, en milieu Safène particulièrement. Une amélioration progressive des systèmes de production s'observe avec la génération de revenus plus importants.

Grâce à ces entreprises, la zone gagne en attractivité. Son essor est notamment lié au développement des périmètres agro-industriels, qui attire davantage de populations allochtones. Cette population vient se greffer aux communautés autochtones, provoquant ainsi des mutations sociales et un développement d'activités connexes. Le transport pour assurer la liaison entre les entreprises, les grands axes routiers (RN1 et RN2) et les lieux de résidence des travailleurs devient un marché prolifique pour des entrepreneurs locaux. La construction de pistes a permis le désenclavement de la plupart des villages. 
Cependant, le revers de la médaille est le fait que les contrats d'acquisition sont de longue durée. L'avenir des générations futures des populations locales n’est pas assuré. Le lien du paysan à la terre est menacé de rupture. La sécurité alimentaire locale et nationale n'est plus assurée, car une grande partie des produits issus des investissements massifs est destinée à l'exportation. Le problème revêt aussi une dimension sociologique puisque les populations sont en train de perdre définitivement leur patrimoine foncier, légué de génération en génération.

\section{Conclusion}

Au début des années 1970, dans le cadre de sa nouvelle politique agricole, l’État du Sénégal avait pris l’option de transiger directement avec les agro-industries pour leur faciliter l'accès à la terre dans la zone pionnière: ce fut le cas avec BUD-Sénégal.

Mais dans le contexte de mondialisation, de globalisation des économies et de décentralisation, ce sont les collectivités locales qui affectent la terre aux agroindustries: c'est le cas avec Soleil Vert, SEMAF, Van Oers, etc. Toutefois, certaines entreprises préfèrent acheter ou louer auprès des propriétaires coutumiers avant de passer à la formalisation avec les institutions compétentes.

Contrairement à la logique de production extravertie accompagnant la réalisation des grands périmètres maraîchers, la plupart des agro-industries cherchent de plus en plus à se positionner sur le marché international tout en "gardant un pied» dans le marché local métropolitain. Cette logique s’inscrit dans l'amélioration du marché dakarois, en pleine émergence économique.

Ce positionnement stratégique sur le marché sénégalais renforce nettement la chaîne de distribution des supermarchés existants dans le pays et entraîne par conséquent l'arrivée des grandes surfaces européennes, dont Auchan. Ce constat conforte l'hypothèse de l'émergence d'une classe moyenne au Sénégal avec, surtout, des exigences sur le respect des normes et la qualité des produits.

Le positionnement des agro-industries dans l'interface ne s'est pas fait ex-nihilo. Il est assujetti à la proximité d'un pôle logistique (AIBD, port minéralier de Guéréo, autoroute à péage et nouveaux corridors, dont Ila Touba et le projet du Train express régional) et d'un marché local, créneau porteur. S’y ajoutent d'autres facteurs stratégiques comme la stabilité politique du pays et la libéralisation croissante de l'économie sénégalaise qui, par ailleurs, favorise le déploiement des agro-industries dans presque toute l'étendue du territoire national.

L'implantation des agro-industries, notamment dans les espaces ruraux, a entraîné des impacts économiques mitigés au plan local. L’essentiel de la main-d'œuvre repose sur des emplois saisonniers précaires (durant les étapes agraires liées au désherbage, à la récolte, au tri, etc.). Ces activités sont, pour la plupart, exercées par la communauté féminine, qui y tire son gagne-pain. Par contre, l'activité agro-industrielle porte atteinte aux ressources naturelles, notamment aux eaux de surface, au foncier (spéculations foncières), ainsi qu'aux espaces boisés affectés par les déclassements abusifs des forêts. 


\section{Bibliographie}

ANAT (AGENCE NATIONALE DE L'AMÉNAGEMENT DU TERRITOIRE RÉPUBLIQUE DU SÉNÉGAL) (2015) Rapport provisoire. Schéma directeur d'aménagement et de développement de la zone DakarThiès-Mbour. Dakar, Ministère de la Gouvernance Locale, du Développement et de l'Aménagement du Territoire.

ANSD (AGENCE NATIONALE DE LA STATISTIQUEET DE LA DÉMOGRAPHIERÉPUBLIQUE DU SÉNÉGAL) (2014) Rapport définitif. Recensement Général de la Population et de l'Habitat, de l'Agriculture et de l'Élevage (RGPHAE) 2013. Dakar, Ministère de l'Économie, des Finances et du Plan.

ARNAUD, Jean-Claude (1970) L'économie maraîchère et fruitière dans la région du Cap-Vert, grande banlieue de Dakar. Strasbourg, Université Louis-Pasteur de Strasbourg, Département de géographie, thèse de doctorat non publiée.

BÂ, Awa (2007) Les fonctions reconnues à l'agriculture intra et périurbaine (AIPU) dans le contexte dakarois : caractérisation, analyse et diagnostic de durabilité de cette agriculture en vue de son intégration dans le projet urbain de Dakar (Sénégal). Paris, AgroParisTech et Dakar, Université Cheikh Anta Diop de Dakar, Sciences agronomiques et environnement, thèse de doctorat non publiée.

BÂ DIAO, Maty (2004) Situation et contraintes des systèmes urbains et périurbains de production horticole et animale dans la région de Dakar. Cahiers Agricultures, vol. 13, n$^{\circ} 1$, p. 39-49.

BELLOT, Jean-Marc et DENIS, Jean-Pierre (1989) Dakar dans les prés de Sangalkam, l'influence de Dakar sur la proche campagne: cas de la communauté rurale de Sangalkam. Dans Pierre Vennetier (dir.) La péri-urbanisation dans les pays tropicaux. Talence, Centre d'Études de Géographie Tropicale et Centre National de la Recherche Scientifique, p. 5-76.
CROUSSE, Bernard, LE BRIS, Émile et LE ROY, Étienne (dir.) (1986) Espaces disputés en Afrique noire: pratiques foncières locales. Paris, Éditions Karthala.

CSE (CENTRE DE SUIVI ÉCOLOGIQUE RÉPUBLIQUE DU SÉNÉGAL) (2014) Données cartographiques. Dakar, Ministère de l'Environnement et du Développement Durable.

DIAGNE, Marius (1990) Sur le phénomène des "jardins du dimanche" dans l'agglomération dakaroise. Dans Émile Le Bris et Hélène Giannitrapani (dir.) Maîtriser le développement urbain en Afrique sub-saharienne. Paris, Office de la recherche scientifique et technique outre-mer, p. 219-226.

DIONE, Diene (1986) Problème de développement des activités du secteur primaire dans la banlieue de Dakar. Limoges, Université de Limoges, Département de géographie, thèse de doctorat non publié.

DIONGUE, Momar (2012) Les périphéries rurales et le métropolisation: mutations et dynamiques territoriales. Le cas de Dakar. Dans Christian Thierry Manga (dir.) Le Sénégal: quelles évolutions territoriales. Paris, L'Harmattan, p. 63-108.

DTGC (DIRECTION DES TRAVAUX G É O G R A P H I Q U E S E T CARTOGRAPHIQUES - RÉPUBLIQUE DU SÉNÉGAL) (2014) Données cartographiques. Dakar, Ministère de l'Équipement et des Transports.

DUBRESSON, Alain (1979) L'espace DakarRufisque en devenir: de l'héritage urbain à la croissance industrielle. Paris, Office de la recherche scientifique et technique outre-mer.

FALL, Safiétou Touré et FALL, Abdou Salam (dir.) (2001) Cités horticoles en sursis? L'agriculture périurbaine dans les grandes niayes au Sénégal. Ottawa, Éditions du Centre de recherches pour le développement international. 
FAYE, Mbaye Mbengue (2007) Ressources végétales de cueillette: transformations récentes dans la communauté rurale de Diass. Dakar, Université Cheikh Anta Diop de Dakar, Département de géographie, mémoire de diplôme d'études approfondies.

GLON, Éric et PECQUEUR, Bernard (2006) Développement et territoires: une question d'environnement et de ressources territoriales. Territoires en mouvement: revue de géographie et aménagement, $\mathrm{n}^{\circ} 1$, p. 13-22.

LELOUP, Fabienne, MOYART, Laurence et PECQUEUR, Bernard (2003) Le développement local en Afrique de l'Ouest: quelle(s) réalité(s) possible(s)? Mondes en développement, vol.4, nº124, p. 95-112.

MBAYE, Alain, DE BON, Hubert et PAGÈS, Jacques (1999) Vers une gestion concertée des ressources naturelles en zone périurbaine. Le cas de la région de Dakar. Dans Paul Moustier, Alain Mbaye et Hubert de Bon (dir.) Agriculture périurbaine en Afrique subsaharienne: actes de l'atelier international du 20 au 24 avril 1998, Montpellier, France. Montpellier, Éditions du Centre de coopération internationale en recherche agronomique pour le développement, p. 125-140.

PECQUEUR, Bernard (2005) Le développement territorial: une nouvelle approche des processus de développement pour les économies du Sud. Dans Benoît Antheaume et Frédéric Giraut (dir.) Le territoire est mort, vive les territoires! Une (re)fabrication au nom du développement. Paris, Éditions de l'Institut de Recherche pour le Développement, p. 295-316.

SÈNE, Louis (2013) Analyse des impacts socio-économiques du périmètre maraîcher Van Oers dans la communauté rurale de Diass. Dakar, Université Cheikh Anta Diop de Dakar, Département de géographie, mémoire de maîtrise non publiée.

THIANDOUM, Mariama (2013) Espace périurbain et activité rurale: dynamiques territoriales en cours en pays safène (Thiès-Sénégal). Dakar, Université Cheikh Anta Diop de Dakar, Département de géographie, thèse de doctorat. 